\title{
Assessment of wound integrity in manual small incision cataract surgery in a tertiary hospital in South India
}

\author{
Ramya M๑, Soumya Ramaniఠ \\ M.S. Ramaiah Medical College, Nagar, Bangalore India
}

\begin{abstract}
BACKGROUND: The study aimed to assess the wound integrity in small incision cataract surgery (SICS) by modified Siedel's test.

MATERIAL AND METHODS: Forty eyes of 40 patients who underwent small incision cataract surgery from a rural background in South India were enrolled in the study after obtaining consent along with the surgical consent from January to March 2020. A complete history was taken, ocular examination and keratometry were performed. B-scan was conducted for patients with mature cataracts. Fundus examination was performed with slit-lamp biomicroscopy and $90 \mathrm{D}$ lens. On the first post-operative day, the best-corrected visual acuity was recorded using the Snellen chart. Anterior segment examination was conducted. Wound integrity was assessed using modified Siedel's test.

RESULTS: In our study, males constituted $55 \%$ of the study population, and females - $45 \%$. The age or sex of the patient did not have a bearing on the need for suture in the patients who underwent small incision cataract surgery $(\mathrm{p}=0.283)$. The cataract grade also did not affect the need for a suture in the study population $(\mathrm{p}=0.280)$. All the patients in our study did not have a positive Seidel's test, notwithstanding the age or sex of the patient, grade of cataract, site of incision, the presence or absence of suture, or the site of the incision, that is superior or superotemporal. CONCLUSION: Manual small incision cataract surgery can be considered a relatively safe and effective procedure for wound integrity and vision improvement.
\end{abstract}

KEY WORDS: small incision cataract surgery (SICS); cataract, modified Siedel's test

Ophthalmol J 2021; Vol. 6, 65-68

\section{INTRODUCTION}

The global burden of blindness due to cataract is estimated to be 53 million, with manual small incision cataract surgery (SICS) playing a significant role in reducing the cataract burden [1]. It is the leading cause of blindness in developing countries [2]. In India, the prevalence of cataracts is about $41 \%$, with the rural burden being $44.6 \%$, and the urban $-43.6 \%$ [3].
In the evolution of cataract surgery, manual SICS is a relatively newer technique than phacoemulsification. Since cost is an essential factor in developing countries, SICS is popular because it is cost-effective and safe, and visual outcome is equivalent to phacoemulsification [4].

Wound construction is an essential step in cataract surgery [5]. In SICS, the scleral wound is constructed in the form of a scleral tunnel inci- 
sion whose characteristics can be customized for each patient. The length, depth, shape, width, and location are important characteristics. Length is planned based on the grade of nuclear sclerosis and the condition of the endothelium. When the grade of the nucleus is higher, the incision has to be more significant to protect the endothelium and avoid inadvertent iris injury while delivering the nucleus.

Similarly, when the corneal endothelium is compromised, the wound should be constructed larger so that easy delivery of the nucleus can occur without damage to the already compromised endothelium. The shape is essential to avoid postoperative astigmatism. The depth of the incision is crucial as too deep an incision can cause uveal tissue prolapse, and too superficial an incision can cause buttonholing of the incision. These factors also determine wound integrity. The width of the incision, which is at least $4 \mathrm{~mm}$, is important to be maintained, as that has a valvular effect, in turn affecting wound integrity. The incision location is to be maintained at an optimal level to prevent iris prolapse. Hence, all the aspects are crucial to maintain wound integrity.

Studies have been done in phacoemulsification, but not many in SCIS, to assess the wound integrity.

In this study, we aim to determine the wound integrity in eyes with superior, superotemporal, and temporal incisions, as well as in patients with and without a side port.

\section{MATERIAL AND METHODS}

Forty patients who underwent small incision cataract surgery in a tertiary hospital in South India were enrolled in the study after obtaining consent and surgical consent. The study adhered to Helsinki's tenets. Complete history, ocular examination, biometry, and keratometry were performed. B-scan was conducted for patients with mature cataracts. Fundus examination was performed with slit-lamp biomicroscopy and $90 \mathrm{D}$ lens.

All patients were subjected to a peribulbar block, with $3.5 \mathrm{~mL} 2 \%$ lignocaine and $2.5 \mathrm{~mL} 0.75 \%$ bupivacaine, with adrenaline 1 in 200,000, and hyaluronidase $50 \mathrm{IU} / \mathrm{mL}$. Under aseptic precautions, a conjunctival peritomy of 3 clock hours was conducted. With the use of bipolar cautery, adequate cautery of episcleral vessels was done. The scleral incision was made using a Bard parker knife with a number 15 blade. The location was based on the surgeon's preference - either superior or superotemporal. The length was based on the grade of the cataract. It was an approximate measure. The shape of the tunnel was a trapezoid. The incision was made such that the inner lip was wider than the outer lip and the length of the inner lip was approximately 2 to $3 \mathrm{~mm}$ more than the outer lip. The inner lip was constructed such that it extended 1 to $2 \mathrm{~mm}$ into the clear cornea to have a valve effect with a tri-planar incision. Then the entry to the anterior chamber was made with a keratome. Continuous curvilinear capsulorrhexis was made either through the main incision or the side port. The wound was extended with a keratome after completing hydro dissection. The nucleus was delivered by sandwich technique after injecting adequate viscoelastic-hydroxypropyl methylcellulose above and below the nucleus. Thorough cortical wash was done with a Simcoe cannula, and a posterior chamber intraocular lens was placed in the bag. The remaining viscoelastic was aspirated. Side port, if made, was hydrated. If the anterior chamber was formed, there was no necessity of suture. In case of a large wound or iris prolapse, the wound was sutured with 9.0 non-absorbable sutures. This was based on the surgeon's decision as well. The eye was closed with a double pad bandage with an ocular shield placed for 18-24 hours.

On the first postoperative day, the best-corrected visual acuity (BCVA) was recorded using a Snellen chart. Anterior segment examination was performed. Wound integrity was assessed in terms of approximation of the wound using a slit-lamp biomicroscope, and wound leak was determined with a modified Seidel's test [6].

Modified Seidel's test was performed by staining the conjunctiva with a fluorescein strip (Fluoro Touch - fluorescein sodium ophthalmic strip USP containing fluorescein sodium IP $1 \mathrm{mg}$ ). Then force was applied at the edge of the incision using a Weck-Cel sponge. Dilution of the dye was looked for using a cobalt blue filter on the slit-lamp biomicroscope, which was considered a positive side test- a wound leak.

\section{RESULTS}

In our study, males constituted $55 \%$ and females $-45 \%$. The age distribution was as shown in Table 1.

Table 2 shows the number of patients with the varying locations of the incision-superior and superotemporal. 


\begin{tabular}{|l|c|c|}
\hline \multicolumn{1}{|l|}{ Table 1. Age distribution } \\
\hline$<50$ years & Frequency & Percent \\
\hline $51-60$ years & 4 & 10.0 \\
\hline $61-70$ years & 11 & 27.5 \\
\hline$>70$ years & 22 & 55.0 \\
\hline
\end{tabular}

\section{Table 2. Site of incision}

\begin{tabular}{|l|c|c|}
\hline Site & Number & Percentage \\
\hline Superior & 14 & $35 \%$ \\
\hline Superotemporal & 26 & $65 \%$ \\
\hline
\end{tabular}

The patient's age did not have a bearing on the need for suture in the patients who underwent small incision cataract surgery $(p=0.283)$. The cataract grade also did not affect the need for a suture in the study population $(\mathrm{p}=0.280)$. All the patients in our study did not have a positive Seidel's test, notwithstanding the age or sex of the patient, grade of cataract, site of incision, or the presence or absence of suture.

Fischer's exact test for the association between the patient's sex and the need for suture was applied with a p-value of 0.310 . The results helped us conclude that the sex of the patient did not have a bearing on the need for a wound suture.

\section{DISCUSSION}

In a study conducted by Singh et al., the prevalence of cataracts was found to be $41 \%$ of the studied population [3]. The prevalence was lower in men than in women. In our study, males constituted $55 \%$ and females $45 \%$.

Small incision cataract surgery is a significant surgery, especially in the setting of rural camps in India. Patients are screened at or near their residence and brought to the base hospital for cataract surgery. Gogate et al. found that only $52.7 \%$ of the patients returned to the base hospital for follow-up [7]. This fact underscores the importance of a well-healed wound and other intraoperative factors to ensure that there is no adverse outcome despite inadequate patient follow-up.

As suggested by Matossian et al., in our study, all patients underwent surgery under a peribulbar block. In addition, ocular bandaging was performed for a period of 18-24 hours after completion of the surgery to ensure no wound leak post-operatively [6].
There are no recent studies in manual SICS as per our literature search regarding wound leak. In our study, though, no wound leak was found in an incision of SICS in a clinical setting.

In a study conducted by Fabiana et al., glue was more effective at preventing wound leakage and bacteria-sized particle influx than other commonly used methods, especially hydrosealing [8]. In our study, the side ports, when made, were sealed using stromal hydration. None of our patients had a post-operative wound leak.

Mallik et al. found that the temporal approach provides a better stabilization of the refraction with a significantly less surgically induced astigmatism than the superior approach [9]. In our literature search, we could not find studies that compared the wound integrity between these incisions in manual SICS.

The limitation of our study is the fact that the incision's size was not objectively measured. In addition, the number of patients was small. Further studies could compare the results of the modified Seidel test in manual SICS and phacoemulsification.

According to a study conducted by Darcy et al., visible aerosol production was found in clear corneal phacoemulsification, especially when the $2.2 \mathrm{~mm}$ tip was brought near the corneal wound [10]. Considering that the incision in a manual SICS is a triplanar incision and that there is no vibrating tip used in the procedure, the probability of aerosol production would be more negligible. In our study, there was no wound leak post-operatively. The vision improvement in a manual SICS in our research and studies conducted by Gogate et al. and Haripriya et al. is comparable to the improvement obtained by phacoemulsification [11]. It has been established that during the COVID-19 pandemic, shortening of surgical time is crucial to reduce the risk of transmission of the disease. This has also been provided as guidance by the Royal College of Ophthalmologists. Singh et al. suggest that the duration of the manual SICS is shorter than phacoemulsification, and vision improvement is similar [12]. Though manual SICS is technically more complex than conventional extracapsular cataract surgery, it provides better functional vision, and as we have seen, no wound leak [13]. In addition, manual SICS has the advantage of being more cost-effective than phacoemulsification [14], maintaining the benefit of good wound integrity. Hence, in the present scenario of COVID-19, quick cataract surgery with less aerosol 
production is the need of the hour. Further studies could help us conclude regarding manual SICS's safety profile compared to phacoemulsification in the current pandemic.

\section{CONCLUSION}

Though the incision in SICS is more significant as compared to phacoemulsification, wound integrity in SICS is well maintained. This was so notwithstanding the size of the nucleus or the site of the incision. Hence, the SICS wound, and therefore the surgery can be considered safe and effective, particularly in those patients where follow-up is difficult such as patients from a rural background. In addition, in the pandemic setting, where faster surgeries, and those that produce lesser aerosols, would be advantageous, SICS can be considered an essential tool in the armamentarium of cataract surgery.

\section{Conflict of interest}

None declared.

\section{REFERENCES}

1. Bernhisel A, Pettey J. Manual small incision cataract surgery. Curr Opin Ophthalmol. 2020; 31(1): 74-79, doi: 10.1097/ ICU.0000000000000624, indexed in Pubmed: 31770166.

2. Liu YC, Wilkins M, Kim T, et al. Cataracts. Lancet. 2017; 390(10094): 600-612, doi: 10.1016/s0140-6736(17)30544-5, indexed in Pubmed: 28242111.

3. Singh $S$, Pardhan $S$, Kulothungan $V$, et al. The prevalence and risk factors for cataract in rural and urban India. Indian J Ophthalmol. 2019; 67(4): 477-483, doi: 10.4103/ijo.IJO_1127_17, indexed in Pubmed: 30900578.

4. Ammous I, Bouayed E, Mabrouk S, et al. [Phacoemulsification versus manual small incision cataract surgery: Anatomic and func- tional results]. J Fr Ophtalmol. 2017; 40(6): 460-466, doi: 10.1016/j. jfo.2017.02.005, indexed in Pubmed: 28576403.

5. Tong AY, Gupta PK, Kim T. Wound closure and tissue adhesives in clear corneal incision cataract surgery. Curr Opin Ophthalmol. 2018; 29(1): 14-18, doi: 10.1097/ICU.0000000000000431, indexed in Pubmed: 28902719.

6. Matossian C, Makari S, Potvin R. Cataract surgery and methods of wound closure: a review. Clin Ophthalmol. 2015; 9: 921-928, doi: 10.2147/0PTH.S83552, indexed in Pubmed: 26045656.

7. Gogate P, Optom JJ, Deshpande $S$, et al. Meta-analysis to Compare the Safety and Efficacy of Manual Small Incision Cataract Surgery and Phacoemulsification. Middle East Afr J Ophthalmol. 2015; 22(3): 362-369, doi: 10.4103/0974-9233.159763, indexed in Pubmed: 26180478.

8. Kashiwabuchi F, Khan Y, Jr MR, et al. Efficacy of three different methods for side port incision wound sealing. Rev Bras Oftalmol. 2013; 72(6): 379-382, doi: 10.1590/s0034-72802013000600005.

9. Mallik VK, Kumar S, Kamboj R, et al. Comparison of astigmatism following manual small incision cataract surgery: superior versus temporal approach. Nepal J Ophthalmol. 2012; 4(1): 54-58, doi: 10.3126/ nepjoph.v4i1.5851, indexed in Pubmed: 22343997.

10. Darcy K, Elhaddad O, Achiron A. Reducing visible aerosol generation during phacoemulsification in the era of Covid-19. Eye (Lond). 2021; 35(5): 1405-1410, doi: 10.1038/s41433-020-1053-3, indexed in Pubmed: 32591733.

11. Haripriya $A$, Chang DF, Reena M, et al. Complication rates of phacoemulsification and manual small-incision cataract surgery at Aravind Eye Hospital. J Cataract Refract Surg. 2012; 38(8): 1360-1369, doi: 10.1016/j.jcrs.2012.04.025, indexed in Pubmed: 22814042.

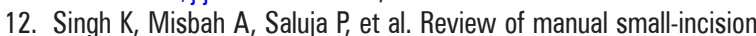
cataract surgery. Indian J Ophthalmol. 2017; 65(12): 1281-1288, doi: 10.4103/ijo.IJO 863 17, indexed in Pubmed: 29208807.

13. Ang M, Evans JR, Mehta JS. Manual small incision cataract surgery (MSICS) with posterior chamber intraocular lens versus extracapsular cataract extraction (ECCE) with posterior chamber intraocular lens for age-related cataract. Cochrane Database Syst Rev. 2014(11): CD008811, doi: 10.1002/14651858.CD008811.pub3, indexed in Pubmed: 25405603.

14. Riaz Y, de Silva SR, Evans JR. Manual small incision cataract surgery (MSICS) with posterior chamber intraocular lens versus phacoemulsification with posterior chamber intraocular lens for age-related cataract. Cochrane Database Syst Rev. 2013(10): CD008813, doi: 10.1002/14651858.CD008813.pub2, indexed in Pubmed: 24114262. 